\title{
Measuring Number of Free Radicals and Evaluating the Purity of Di(phenyl)-(2,4,6-trinitrophenyl)iminoazanium [DPPH] Reagents by Effective Magnetic Moment Method
}

\author{
Nobuhiro MATSUMoto ${ }^{\dagger}$ and Nobuyasu IтоH \\ National Metrology Institute of Japan (NMIJ), National Institute of Advanced Industrial Science and Technology \\ (AIST), AIST Central-3, 1-1-1 Umezono, Tsukuba, Ibaraki 305-8563, Japan
}

\begin{abstract}
Di(phenyl)-(2,4,6-trinitrophenyl)iminoazanium [DPPH] is widely used as a standard for measuring the number of free radicals. Here, we evaluated the number of free radicals of "DPPH" reagents from three manufacturers by effective magnetic moment method. Interestingly, the reagents from different manufacturers had varying temperature dependencies for both magnetic moment and $g$-value at low temperatures. As a result, the maximum relative difference among the three reagents on the number of free radicals per unit mass was 20\%. Carbon hydrogen nitrogen (CHN) analyses, highresolution EPR measurements, FT-IR measurement, and NMR measurement confirmed that a major component of only one among the three reagents was "pure" DPPH. The evaluated purity based on free radical content was $0.998 \mathrm{~kg} \mathrm{~kg}^{-1}$ with expanded uncertainty of $0.036 \mathrm{~kg} \mathrm{~kg}^{-1}$. The other two reagents were found to be contaminated by several $\%$ of benzene in the DPPH crystal structure.
\end{abstract}

Keywords Primary direct method for amount of substance, Curie-Weiss law, EPR, SQUID, free radical analysis, standard sample, singlet state, low temperature measurement

(Received March 16, 2018; Accepted April 27, 2018; Published August 10, 2018)

\section{Introduction}

Purity is a fundamental quality for various chemicals and has an important bearing on the accuracy attained by any analysis. ${ }^{1}$ The primarily direct method for measuring the amount of a substance is a kind of absolute quantitative analytical method and can measure the fraction of unknown substances without reference to a standard. Thus, purity is preferably determined by "potential primary direct methods of measurement for the amount of substance", ${ }^{2}$ such as gravimetry, coulometry, and freezing point depression method.

Here, we propose a quantitative analytical method using effective magnetic moment, ${ }^{3-7}$ based on the combination of the Curie-Weiss law and the fundamental equation of electron paramagnetic resonance [EPR]. The "effective magnetic moment method" measures the number of atoms (quantum free spins) with unpaired electrons (such as organic free radical) that exist in a diamagnetic matrix. A superconducting quantum interference device [SQUID] and an EPR spectrometer are used for measurements of magnetic moment and $g$-values, respectively. Previously, ${ }^{5,6}$ the effective magnetic moment method was applied for purity analyses of nitroxyl powders of 2,2,6,6-tetramethylpiperidine [TEMPO], 4-hydroxy-2,2,6,6tetramethyl piperidine [TEMPOL], and 4-hydroxy-2,2,6,6tetramethyl piperidine 1-oxyl [4HTB]. The study revealed that the purities of TEMPO and 4HTB based on their free radical

$\dagger$ To whom correspondence should be addressed.

E-mail: nobu-matsumoto@aist.go.jp content were approximately $1 \mathrm{~kg} \mathrm{~kg}^{-1}$ with relative expanded uncertainty of $1 \%$.

Owing to its stable nitrogen radical, di(phenyl)-(2,4,6trinitrophenyl)iminoazanium [DPPH, 2,2-diphenyl-1-picrylhydrazyl, or, 1,1-diphenyl-2-picrylhydrazyl free radical] is commonly utilized in analytical chemistry to evaluate antioxidant activity with the aid of UV/visible photometers, EPR spectrometers, or other analytical instruments. ${ }^{8-16}$ The reagents of DPPH are used as a standard for free radical content and other applications in titration, ${ }^{17}$ dating for geometry, ${ }^{18}$ solar cell, ${ }^{19}$ and medical diagnosis. ${ }^{20}$ Furthermore, DPPH has been also used as a standard of sweep field on EPR spectroscopy, ${ }^{21,22}$ or as a test sample, for developing EPR-based measurement techniques in biochemistry, ${ }^{23}$ solid state physics, ${ }^{24,25}$ geometry, ${ }^{26,27}$ and others. ${ }^{28-30}$

When practitioners of EPR calculate the number of free radicals in a DPPH reagent, they use the purity value provided by the manufacturer confirmed by either subtraction method, ${ }^{30}$ or area normalization method. ${ }^{1}$ A common problem in both methods is that not all impurities can be detected and quantified. However, the effective magnetic moment method can evaluate accurate purity based on free radical content.

In this study, we applied the effective magnetic moment method to evaluate the free radicals, effectively quantifying the purity of DPPH reagents from three different manufacturers. Results were compared with each other. Interestingly, the SQUID magnetometry and EPR spectroscopy using an X-band microwave (approx. 9.4 GHz) for each reagent showed significantly different temperature dependencies for both magnetic moment and $g$-value, nevertheless the DPPH has been 
one of the representative reagents with a long history and many researchers have already reported the physical and chemical properties of pure DPPH, DPPH-derivatives, and DPPHcomplexes. $^{31-43}$ Elemental analysis, high-resolution EPR spectroscopy using higher-frequency microwaves of Q-(approx. $34 \mathrm{GHz}$ ) and W-bands (approx. $94 \mathrm{GHz}$ ), FT-IR spectroscopy and NMR spectroscopy were also performed to identify the composition of each reagent. These revealed that only one reagent among the three was exactly pure. Its purity based on free radical content was also obtained. The possibility of free radical impurities in the pure DPPH reagent will be discussed, based on the results of SQUID and X-band EPR measurements at low temperatures slightly below liquid helium (He) temperature.

\section{Experimental}

\section{DPPH reagents}

The reagents sold as "pure DPPH" powder were purchased from three different manufactures. They will be denoted as "reagent A," "reagent B," and "reagent C," accordingly. Two of the manufacturers provided purity information determined by some methods.

\section{Magnetic moment measurement}

Magnetic moment measurements at above $4.2 \mathrm{~K}$ were obtained using a SQUID magnetometer (Quantum Design, MPMS-7 DC magnetometer with horizontal rotator option) under DC measurement mode with a scan length of $4 \mathrm{~cm}$. The preparation method of the sample was adopted from our previous paper. ${ }^{6}$ The total mass of the sample, $W$, was measured using an analytical balance with a minimum readability of $0.01 \mathrm{mg}$. Another SQUID magnetometer (MPMS-5 DC magnetometer with RSO option) under RSO measurement mode was utilized at low temperatures slightly below liquid helium temperature.

\section{EPR spectroscopy for g-value measurement}

An EPR spectrometer (Bruker, EMX) was used with a cryostat (Oxford Instruments, ESR900) for the measurement at X-band. Liquid $\mathrm{He}$ was used as cryogen for the sample. A powder sample of approximately $1 \mathrm{mg}$ was sealed in an evacuated quartz-Pyrex tube (diameter and length of quartz part were 4 $\mathrm{mm}$ and $100 \mathrm{~mm}$, respectively). He gas below atmospheric pressure was also introduced into the tubes because of its good thermal conductivity. The E500 was also used with a cryostat (Oxford Instruments, ESR910) for X-band EPR measurement at temperatures lower than liquid He temperature.

\section{CHN analysis, and high-frequency EPR, FT-IR, and NMR} spectroscopy

A 2400 Series II CHNS/O System elemental analyzer (PerkinElmer, Waltham, MA, USA) and an ultra-micro balance MC5 (Sartorius, Göttingen, Germany, weighing range of $0.1 \mu \mathrm{g}$ to $2.1 \mathrm{~g}$ ) were used for the carbon hydrogen nitrogen $(\mathrm{CHN})$ analysis. $\quad N$-Phenylacetamide (acetanilide, $\mathrm{C}_{8} \mathrm{H}_{9} \mathrm{NO}$ ) obtained from PerkinElmer was used as a calibrant. The experimental procedures reported by previous studies were followed. ${ }^{44,45}$

To obtain EPR spectra with higher resolution than those observed at X-band, EPR spectrometers (Bruker, E500 and E680) were operated at Q- and W-bands EPR, respectively, to obtain considerably higher resolution EPR spectra than those of X-band. A powdered sample for the Q-band of less than $1 \mathrm{mg}$ was sealed in an evacuated quartz tube (diameter $2 \mathrm{~mm}$, length $100 \mathrm{~mm}$ ). He gas, below atmospheric pressure, was introduced

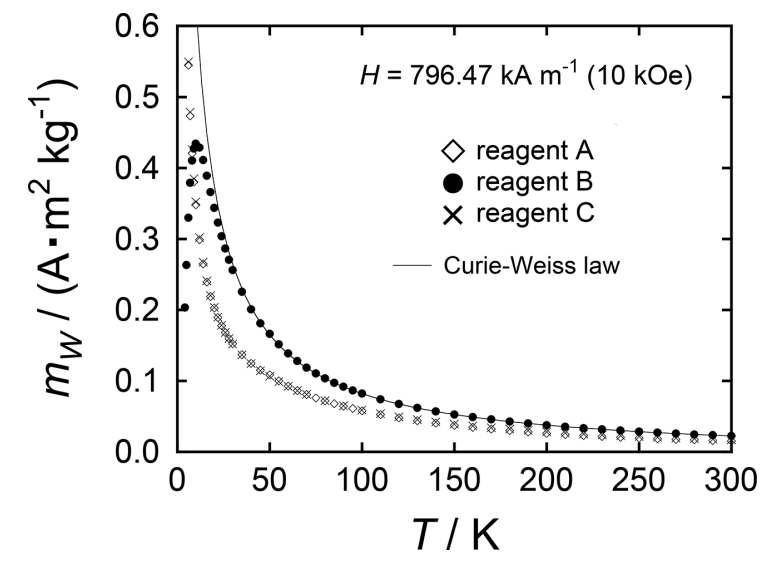

Fig. 1 Temperature dependence of magnetic moment per unit mass, $m_{\mathrm{W}}$, under a magnetic field, $H$, of $796.38 \mathrm{kA} \mathrm{m}^{-1}$ (corresponding to 1 tesla) over a temperature range of $4.2 \mathrm{~K}$ to $300 \mathrm{~K}$. The $m_{\mathrm{W}}$ equivalent to $m$ divided by $W$, where the $m$ is magnetic moment measurement from SQUID and the $W$ is the mass of the sample measured with an electronic balance. The $W$ of the samples were $10.22 \pm 0.06 \mathrm{mg}$, $17.00 \pm 0.06 \mathrm{mg}$ and $17.14 \pm 0.06 \mathrm{mg}$, for reagents $\mathrm{A}, \mathrm{B}$, and $\mathrm{C}$, respectively. The solid line represents the theoretical curve according to the Curie-Weiss law and is observably fitted to the data of reagent B of 55 to $300 \mathrm{~K}$.

into the tubes. For the W-band measurements at room temperature, a cryostat and superconducting magnet were used. A sample powder of each reagent was sealed in a quartz tube (diameter $0.9 \mathrm{~mm}$, length $100 \mathrm{~mm}$ ).

Mid-infrared spectra ranging from 4000 to $400 \mathrm{~cm}^{-1}$ were measured by an FT-IR spectroscopy (Perkin Elmer, SPECTRUM2000). A potassium bromide (KBr) pellet with a diameter of $7 \mathrm{~mm}$ including sample powder was made using $\mathrm{KBr}$ crystal blocks and a table press. Far-infrared spectra less than $400 \mathrm{~cm}^{-1}$ were also obtained by an FT-IR spectroscopy (Bruker, IFS66v/S). Beads of cesium iodide (Sigma-Aldrich) was used for pellet preparation.

${ }^{1} \mathrm{H}$ NMR and ${ }^{13} \mathrm{C}$ NMR spectroscopies were performed using an Avance III 500 spectrometer (Bruker Biospin, Rheinstetten, Germany; $500.13 \mathrm{MHz}$ for ${ }^{1} \mathrm{H}$ ), to detect impurities without any free radical. Approximately $50 \mathrm{mg}$ powder of each reagent was dissolved in dimethyl sulfoxide- $d_{6}$ [DMSO- $\left.d_{6}\right]$ with tetramethylsilane [TMS].

\section{Results and Discussion}

\section{SQUID and X-band EPR measurements}

Figure 1 shows the temperature dependencies of magnetic moment per unit mass, $m_{\mathrm{W}}$, for the different reagents measured by the SQUID magnetometer. The magnetic moment of reagent $\mathrm{B}$ behaves in accordance with the Curie-Weiss law behavior from 300 to $60 \mathrm{~K}$, but not below $50 \mathrm{~K}$. Additionally, a maximum value appears at $10 \mathrm{~K}$, but then rapidly decreases. Pure DPPH powder by Fujito, ${ }^{40}$ and a DPPH single crystal by Žilić ${ }^{43}$ exhibit this similar temperature dependence. The behavior of reagent $B$ at lower temperatures will be mentioned in the later subsection. On the other hand, magnetic moments for both reagents A and $\mathrm{C}$ appear to be linearly proportional to the inverse temperature, $T^{-1}$, over a wide temperature range of 4.2 to $300 \mathrm{~K}$.

The Curie-Weiss law is expressed by the equation,

$$
m=c_{\text {Curie }}(T-\Theta)^{-1}+m_{0},
$$




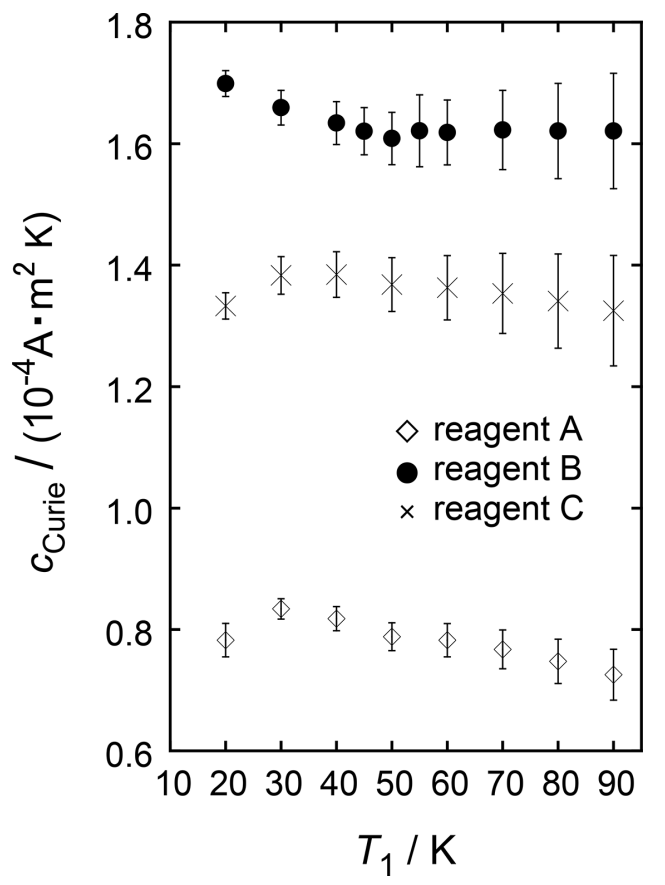

Fig. 2 A parameter analogous to Curie constant, $c_{\text {Curie, }}$ when analytical temperature range for the data set of $m$ and $T$ is between $T_{1}$ and $T_{2}$. The values of $T_{2}$ for all the reagents are fixed at $300 \mathrm{~K}$.

where $m$ is the magnetic moment, $\Theta$ is the Weiss temperature, $m_{0}$ is the diamagnetic magnetic moment owing to all closed electrons in both the sample and background, and $c_{\text {Curie }}$ is analogous to Curie's constant. Figure 2 shows the $c_{\text {Curie }}$ values when non-linear regression analysis was performed following our previous procedure ${ }^{6}$ at a temperature range of $T_{1}-T_{2}$, where $T_{2}$ is fixed at $300 \mathrm{~K}$. The constant is associated with the number of free radicals in each sample, $n$. Figure S1 (Supporting Information) shows the modified residual sum of the weighted squared deviation, $S_{\text {res, }}$ related to Deming's least-squares method, corresponding to the analytical temperature range of Fig. 2. For reagent $\mathrm{B}$, the values of $c_{\text {Curie }}$ are almost constant over the $T_{1}$ range of 55 to $90 \mathrm{~K}$. However, the expanded uncertainty (error bar) of the $c_{\text {Curie }}$ observably decreases with $T_{1}$. The $c_{\text {Curie }}, \Theta$, and $m_{0}$ are $(1.621 \pm 0.060) \times 10^{-4} \mathrm{~A} \mathrm{~m}^{2} \mathrm{~K}$, $-(4.8 \pm 2.5) \mathrm{K}$, and $(-1.51 \pm 0.18) \times 10^{-7} \mathrm{~A} \mathrm{~m}^{2}$ at $T_{1}=55 \mathrm{~K}$. In contrast, the $c_{\text {Curie }}$ values for both reagents $\mathrm{A}$ and $\mathrm{C}$ vary within the same range. Moreover, their appropriate analytical temperature ranges appear difficult to determine. The relative differences of $c_{\text {Curie }}$ between $T_{1}=50 \mathrm{~K}$ and $T_{1}=90 \mathrm{~K}$ are $10 \%$ and $4 \%$ for reagents $\mathrm{A}$ and $\mathrm{C}$, respectively. Furthermore, the $\Theta$ of reagents $A$ and $C$ at $T_{1}=50 \mathrm{~K}$ are $-15 \pm 2 \mathrm{~K}$ and $-20 \pm 2 \mathrm{~K}$, respectively. Accordingly, absolute values of these are greater than that from reagent B. A negative Weiss temperature generally reflects the magnitude of antiferromagnetic interaction between magnetic species.

For the X-band EPR spectroscopy, only a single first-derivative peak was detected in each sample. Figure 3 illustrates temperature dependencies of the effective $g$-value, $g_{\text {eff }},{ }^{6}$ at $\mathrm{X}$-band microwave. Each $g_{\text {eff }}$ means non-corrected $g$-value and was simply obtained by substituting microwave frequency and magnetic field (when magnetic resonance occurs) in the EPR fundamental equation. The $g_{\text {eff }}$ near room temperature for each sample was within $2.0036 \pm 0.0002$, values which are reported in a database. ${ }^{46}$ However, below $50 \mathrm{~K}$, there are apparent

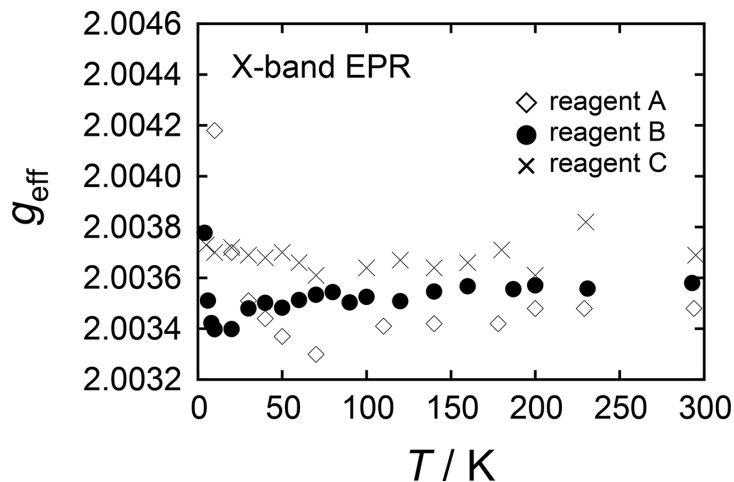

Fig. 3 Effective $g$-value, $g_{\text {eff }}$, from the X-band EPR measurements, over a temperature range of $10 \mathrm{~K}$ to room temperature.

Table 1 Results of CHN analyses (unit of each value is wt $\%$ )

\begin{tabular}{ccccc}
\hline $\begin{array}{c}\text { Theoretical } \\
\text { value }\end{array}$ & $\mathrm{C}$ & $\mathrm{H}$ & $\mathrm{N}$ & $\mathrm{O}^{\mathrm{b}}$ \\
\cline { 2 - 5 } & 54.8 & 3.1 & 17.8 & 24.3 \\
\hline Reagent A & $55.4[0.5]$ & $3.2[0.2]$ & $16.7[-1.1]$ & $24.7[0.4]$ \\
Reagent B & $54.7[-0.1]$ & $3.0[0.0]$ & $17.6[-0.2]$ & $24.7[0.3]$ \\
Reagent C & $55.3[0.5]$ & $3.1[0.1]$ & $16.1[-1.7]$ & $25.5[1.2]$ \\
\hline
\end{tabular}

a. The theoretical value is a value corresponding to stoichiometric composition of DPPH, $\mathrm{C}_{18} \mathrm{H}_{12} \mathrm{~N}_{5} \mathrm{O}_{6}$. The values in brackets are the corresponding difference between the theoretical value and experimental value.

b. Each value of $\mathrm{O}$ is the sum of $\mathrm{C}, \mathrm{H}$ and $\mathrm{N}$ subtracted from $100 \mathrm{wt} \%$.

differences among the three samples. Figure S2(a) (Supporting Information) shows the line-width of the first-derivative peak, $W_{\mathrm{pp}}$, above $10 \mathrm{~K}$. The $W_{\mathrm{pp}}$ of reagent B is the smallest over the entire temperature range. The line shapes representing the $W_{\mathrm{pp}} / W_{\mathrm{hh}}$ ratio of reagent $\mathrm{B}$ between $50 \mathrm{~K}$ and room temperature follows the Lorentzian function as shown in Fig. S2(b) (Supporting Information). ${ }^{47-49} \quad W_{\mathrm{hh}}$ is half bandwidth of the absorption line. Furthermore, the line shapes of reagents A and the $\mathrm{C}$ at room temperature deviate from the Lorentzian function. The $g$-value, $g$, of reagent $\mathrm{B}$, which will be used for calculation of purity based on free radical content, is estimated at $2.0036 \pm 0.0001$. The standard deviation over a temperature range of $50 \mathrm{~K}$ to room temperature was obtained by considering the small difference of $g_{\text {eff }}$ between the up and down direction of the magnetic field sweep. ${ }^{6}$ The value of $g$ at $297 \mathrm{~K}$ of reagent B was also observed by Žilić. ${ }^{43}$

From the above results of the $c_{\text {Curie }}, H, g$ and $W$, the number of free radicals per unit mass for each sample were $(1.23 \pm 0.04) \times$ $10^{24} \mathrm{~kg}^{-1}$ for reagent $\mathrm{A},(1.524 \pm 0.056) \times 10^{24} \mathrm{~kg}^{-1}$ for reagent $\mathrm{B}$ and $(1.28 \pm 0.04) \times 10^{24} \mathrm{~kg}^{-1}$ for reagent $\mathrm{C}$, respectively. The maximum relative difference among the three reagents on the number of free radicals per unit mass was $20 \%$.

CHN analysis, high-frequency EPR, FT-IR and NMR measurement

The observed magnetic properties show that the qualities of the reagents vary, suggesting differences in their main components. The results of the CHN analyses were performed for each reagent sample are shown in Table 1. Only reagent B has contents of $\mathrm{C}, \mathrm{H}$, and $\mathrm{N}$ corresponding to the theoretical composition of the DPPH. The general accuracy is within 


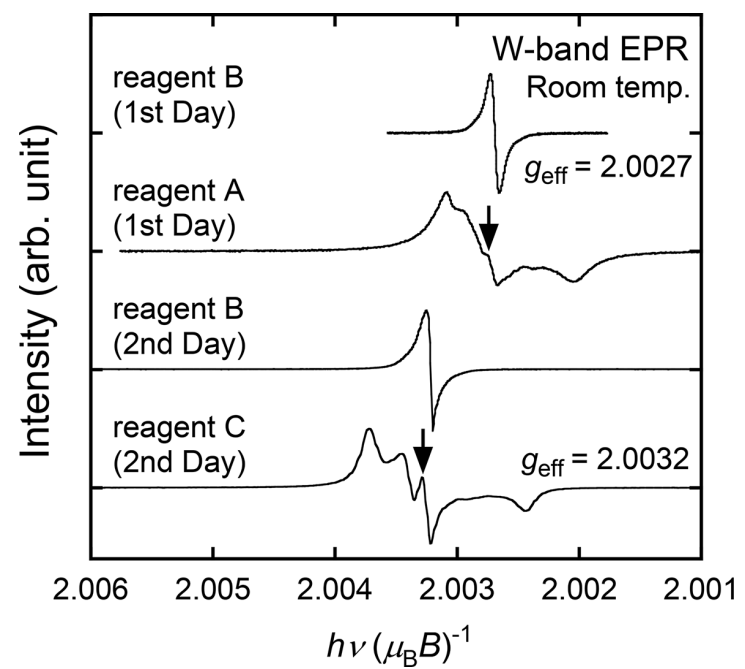

Fig. 4 W-band EPR spectra at room temperature. The $\mu_{\mathrm{B}}, B, h$, and $v$ in horizontal axis correspond to the Bohr magneton, magnetic field, plank constant, and microwave frequency, respectively. The peaks that the arrows indicate pertain to the same peaks as that of reagent B.

$\pm 0.3 \mathrm{wt} \%$, comparable to that of a single crystal of pure DPPH by Žilić which had a deviation of $-0.35 \mathrm{wt} \%$ for C. ${ }^{43}$ For both reagents $\mathrm{A}$ and $\mathrm{C}$, the carbon content was slightly higher than the theoretical value, while the $\mathrm{N}$ content was approximately $1 \mathrm{wt} \%$ less than that of the theoretical content.

Figure S3 (Supporting Information) shows the Q-band EPR spectra at room temperature for all reagents. The spectrum of reagent $\mathrm{B}$ shows a single first-derivative peak, similar to that from the X-band. However, the spectra of reagents A and C exhibited a three-axial $g$ anisotropy. This and two-axial $g$ anisotropy were reported in DPPH-derivatives or "complexes" (compounds that where solvent molecules that existed in the DPPH crystal structure). ${ }^{43,46}$ In addition, some researchers reported that polycrystalline DPPH retains the isotropic single peak even at very high-frequencies of 108.8 and $464.8 \mathrm{GHz}{ }^{21,22}$ Figure 4 shows the W-band (approx. $94 \mathrm{GHz}$ ) EPR spectra at room temperature on separate days. Reagent B showed an isotropic first-derivative peak on both days with slightly different $g_{\text {eff }}$ values. Because their $g_{\text {eff }}$ values were even smaller than that of the X-band as mentioned above, the $g_{\text {eff }}$ of the W-band is not considered reliable for discussion and it partly attributed to slight differences in the instrument's conditions. As for the peak shape, both reagents $\mathrm{A}$ and $\mathrm{C}$ have an additional peak that appears at the same position as the isotropic peak of reagent $B$, in addition to the anisotropic peaks detected in the Q-band of Fig. S3. The height of each additional peak is smaller than that of the anisotropic peak.

Figure S4 (Supporting Information) illustrates the mid-IR spectra of each reagent at room temperature. The spectrum of reagent $\mathrm{B}$ is the same as that of DPPH from the database, ${ }^{50}$ while the spectra of reagents $\mathrm{A}$ and $\mathrm{C}$ resemble each other. However, there are apparent differences within the wavelength regions of $1280-1350$ and $1490-1610 \mathrm{~cm}^{-1}$ between reagent $\mathrm{B}$ and the reference, and reagents $\mathrm{A}$ and $\mathrm{C}$, respectively. ${ }^{43,51}$

Figure S5 (Supporting Information) displays the NMR spectra for impurity analysis when the powder samples were dissolved in DMSO- $d_{6}$. A detailed explanation of this figure is provided in the caption. Importantly, benzene $\left(\mathrm{C}_{6} \mathrm{H}_{6}\right)$ of several mol\% was detected in both reagents $\mathrm{A}$ and $\mathrm{C}$, but not in reagent $\mathrm{B}$.

The starting materials for the synthesis of DPPH are $\mathrm{DPPH}_{2}$, lead oxide $(\mathrm{PbO})$, and a solvent. Examples of the solvent such as benzene, diethyl ether, carbon disulfide, carbon tetrachloride $\left(\mathrm{CCl}_{4}\right)$, and chloroform, and others were reported and their solvents were also used for purification of synthesized products. ${ }^{37,40,42,43,52}$ It was reported that synthesis of pure DPPH were successfully achieved when the solvent was either diethyl ether, ${ }^{37}$ or ether. ${ }^{43}$ One of the starting materials, $\mathrm{PbO}$ was not detected in the far-IR spectrum. ${ }^{53}$ The NMR spectra of reagents $A$ and $C$ suggest that benzene was used as their synthesis solvent. The detected benzene molecules may exist in the crystal structure of DPPH and were eluted to DMSO- $d_{6}$ when the powder samples were dissolved in the DMSO- $d_{6}$. The anisotropic peaks in both Q-band and W-band EPR spectra may be attributed to this. Fujito illustrated a crystal structure of a complex when the ratio of DPPH to benzene was 1 to 1 [DPPHbenzene complex]. ${ }^{40}$ Based on the result of CHN analyses in Table 1, the $\mathrm{C}$ and $\mathrm{H}$ contents of reagents $\mathrm{A}$ and $\mathrm{C}$ were considerably larger than theoretical values. Assuming that a DPPH $\left(\mathrm{C}_{18} \mathrm{H}_{12} \mathrm{~N}_{5} \mathrm{O}_{6}\right)$ and a benzene $\left(\mathrm{C}_{6} \mathrm{H}_{6}\right)$ molecule both exist with a molar ratio of 0.93 to 0.07 , the $\mathrm{C}$ and $\mathrm{H}$ contents of the latter reagents would satisfy theoretical values. Accordingly, the experimental $\mathrm{N}$ content would adjust to 0.8 and $1.5 \mathrm{wt} \%$ for reagents $\mathrm{A}$ and $\mathrm{C}$ smaller than the theoretical values. However, all results of qualitative analyses cannot completely explain the results of the $\mathrm{CHN}$ analyses.

Representative results by effective magnetic moment method and other instrumental analytical methods

Table 2 summarizes the number of free radicals per unit mass, $n_{\mathrm{w}}$, and temperature range obeying the Cuire-Weiss law and the Weiss temperature, which were obtained from both the SQUID and X-band EPR measurements. The number of free radicals per unit mass, $n_{\mathrm{w}}$, for reagents $\mathrm{A}$ and $\mathrm{C}$ were similar, and were $20 \%$ less than that for reagent B. The uncertainty is about 3 times larger than what was obtained for TEMPO and the 4HTB in our previous work. ${ }^{6}$ This is attributed to the higher $T_{1}$ value of reagent $\mathrm{B}$ that obeyed the Curie-Weiss law, in comparison with a $T_{1}$ of $10 \mathrm{~K}$ for both the TEMPO and the 4HTB.

The peak shape on the Q- and W-EPR spectrum, the Weiss temperature, and major component for each reagent are also summarized in this table. Since the qualitative analyses confirmed that only reagent $\mathrm{B}$ was pure DPPH, its purity in terms of free radical content can be obtained using a formula as shown in Table 2. Consequently, the purity for the sample was calculated at $0.998 \mathrm{~kg} \mathrm{~kg}^{-1}$ within its expanded uncertainty of $0.036 \mathrm{~kg} \mathrm{~kg}^{-1}$.

Magnetic behaviors at low temperatures and possibility of impurities with free radicals in reagent $B$

The presence of other paramagnetic compounds as impurities in the sample of reagent $\mathrm{B}$ and their possible manifestation as free radicals were investigated. Based on the results of higher resolution (the Q- and W-bands) EPR measurements at room temperature, and SQUID and X-band EPR measurements below liquid He temperature, as shown in Figs. 5 and 6, respectively, the possibility of such is low.

For reagent B, Fig. 5 shows the temperature dependence of $m$ from the SQUID measurement at 1.8 to $12 \mathrm{~K}$. Its magnetic moment deviates from the Curie-Weiss law below $50 \mathrm{~K}$ and rapidly decreases below $10 \mathrm{~K}$. This phenomenon is caused by the isolated pair model, ${ }^{40}$ where nitrogen radicals of two DPPH molecules make a singlet-pair (total spin quantum number, $S$ is 0 ) at low temperatures. The orientations of the two quantum spins in each singlet pair is anti-parallel and no interaction exists among the singlet pairs. The following Eq. (2) of the 
Table 2 Representative analytical results for each reagent

\begin{tabular}{|c|c|c|c|c|c|c|}
\hline Reagent & $\begin{array}{c}\text { Temp. range } \\
\text { obeying Curie-Weiss } \\
\text { law }\end{array}$ & $\begin{array}{l}\text { Number of free } \\
\text { radicals per unit } \\
\text { mass, } n_{\mathrm{W}^{a} / \mathrm{kg}^{-1}}\end{array}$ & $\begin{array}{l}\text { Weiss } \\
\text { temp., } \\
\Theta / \mathrm{K}\end{array}$ & $\begin{array}{l}\text { Peak shape } \\
\text { in Q- and } \\
\text { W-EPR }\end{array}$ & $\begin{array}{c}\text { Major } \\
\text { component }\end{array}$ & $\begin{array}{l}\text { Purity as free } \\
\text { radical, } \\
x_{\text {anal }}^{\text {a,c }} / \mathrm{kg} \mathrm{kg}^{-1}\end{array}$ \\
\hline A & $\begin{array}{l}50 \text { to } 300 \mathrm{~K} \text { (monotonous } \\
\text { increase more than } 4.2 \mathrm{~K} \text { ) }\end{array}$ & $(1.23 \pm 0.04) \times 10^{24 b}$ & $-15 \pm 2$ & Anisotropic & $\begin{array}{l}\text { DPPH contaminated by } \\
\text { several mol\% of } \mathrm{C}_{6} \mathrm{H}_{6}\end{array}$ & - \\
\hline B & $\begin{array}{l}55 \text { to } 300 \mathrm{~K} \text { (singlet-state } \\
\text { below around } 10 \mathrm{~K} \text { ) }\end{array}$ & $(1.524 \pm 0.056) \times 10^{24}$ & $-4.8 \pm 2.5$ & Isotropic & $\mathrm{DPPH}$ & $0.998 \pm 0.036$ \\
\hline $\mathrm{C}$ & $\begin{array}{l}50 \text { to } 300 \mathrm{~K} \text { (monotonous } \\
\text { increase more than } 4.2 \mathrm{~K} \text { ) }\end{array}$ & $(1.28 \pm 0.04) \times 10^{24 b}$ & $-20 \pm 2$ & Anisotropic & $\begin{array}{l}\text { DPPH contaminated by } \\
\text { several } \mathrm{mol} \% \text { of } \mathrm{C}_{6} \mathrm{H}_{6}\end{array}$ & - \\
\hline
\end{tabular}

a. Each value of $n_{\mathrm{W}}$ and $x_{\text {anal }}$ is accompanied by its expanded uncertainty (with a coverage factor of 2). $\mathrm{b}$. These $n_{\mathrm{W}}$ values are significantly dependent on the analytical temperature range ( $T_{1}$ to $T_{2}$ ) of the data set of $m$ and $T$. In this table, the results are shown when $T_{1}$ is $50 \mathrm{~K}$ and $T_{2}$ is $300 \mathrm{~K}$. Both $T_{1}$ and $T_{2}$ were regarded as appropriate analytical temperature boundaries, based on the results of Fig. S1. c. The $x_{\text {anal }}$ is defined as the following formula; $x_{\text {anal }}=n_{\mathrm{W}}$ (molar mass of major component) $/[($ number of radical per molecule)(Avogadro's number) $]$.

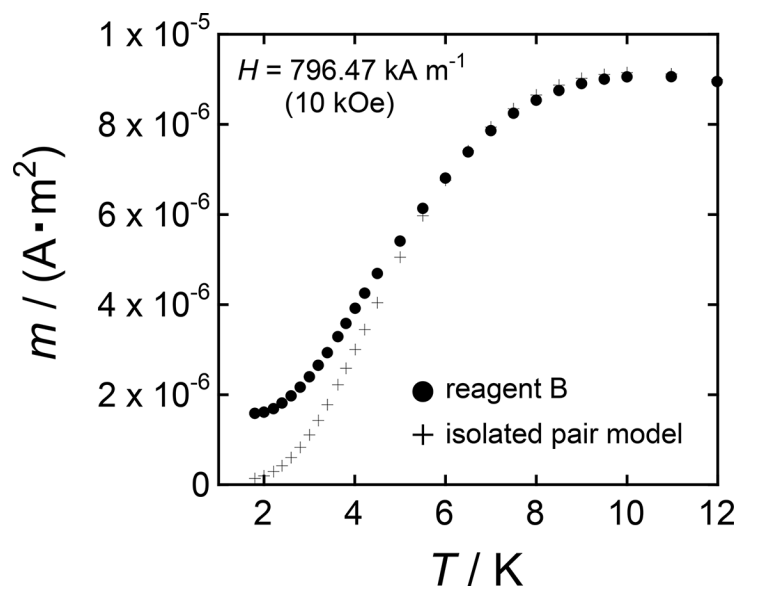

Fig. 5 Temperature dependence of magnetic moment for reagent $\mathrm{B}$ slightly below liquid He temperature, under $H$ of $796.38 \mathrm{kA} \mathrm{m}^{-1}$. The mass of this sample was $20.73 \pm 0.04 \mathrm{mg}$. The theoretical curve is also plotted according to the isolated pair model when the parameters in Eq. (2) are $7.6266 \times 10^{-4} \mathrm{~A} \mathrm{~m}^{2} \mathrm{~K}$ for $2 n^{\prime} g^{2} \mu_{0} \mu_{\mathrm{B}^{\prime}}{ }^{2} k_{\mathrm{B}}{ }^{-1}, 4.3 \times 10^{-8} \mathrm{~A} \mathrm{~m}^{2}$ for $m_{0}$, and $17 \mathrm{~K}$ for $\delta / k_{\mathrm{B}}$. The experimental result above $6 \mathrm{~K}$ approximates the theoretical curve.

isolated pair model roughly describes temperature dependence of $m$ at low temperatures near liquid He temperature: ${ }^{40}$

$$
m=2 n^{\prime} g^{2} \mu_{0} \mu_{\mathrm{B}}^{\prime}\left[k_{\mathrm{B}} T\left(3+\exp \left(-J / k_{\mathrm{B}} T\right)\right]^{-1} H+m_{0},\right.
$$

where the $\mu_{0}, k_{\mathrm{B}}$ and $\mu_{\mathrm{B}}$ are the magnetic permeability of free space, the Boltzmann constant and the Bohr magneton, respectively. The $n^{\prime}$ is the number of the nitrogen radical pair and equals $0.5 n$. The $J$ is Heisenberg exchange coupling between the two unpaired electrons. The higher temperature approximation gives the same equation as Eq. (1) based on the Curie-Weiss law. At temperatures below $-J / k_{\mathrm{B}}$ (in this case, approx. $10 \mathrm{~K}), m$ rapidly decreases with decreasing $T$, and approaches $m_{0}$ as $T$ approaches $0 \mathrm{~K}$, then $m$ approaches $m_{0}$ when $T$ approaches $0 \mathrm{~K}$. In Fig. 5, the observed magnetic moment is comparable to the theoretical curve above around $6 \mathrm{~K}$ and then the theoretical curve decreases to zero at around $1.8 \mathrm{~K}$.

Fujito reported that the magnetic susceptibility of his pure DPPH powder between 2.5 and $1.8 \mathrm{~K}$ increased slightly with decreasing $T$ and concluded that this increase was caused by the Curie-Weiss law effect of isolated paramagnetic impurities with

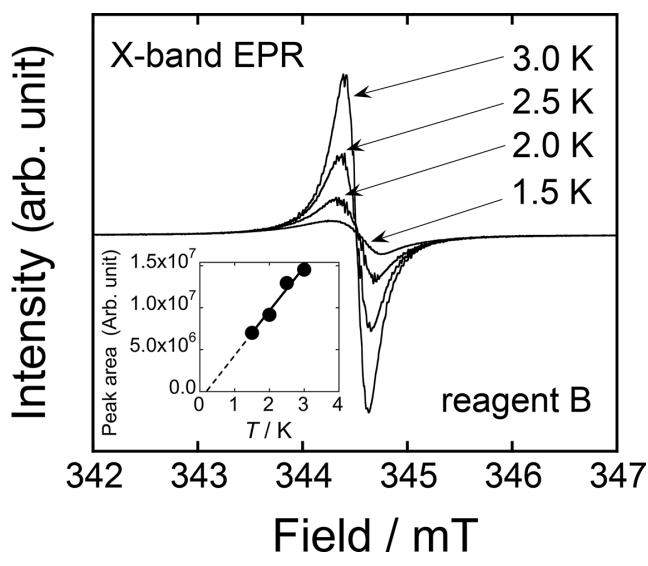

Fig. 6 X-band EPR spectrum of reagent B over a temperature of 1.5 to $3 \mathrm{~K}$.

"spin concentration" of $2.5 \% .^{40}$ The magnetic susceptibility of DPPH single crystal was reported by Žilić to slightly increased near $0 \mathrm{~K}^{43}$ In contrast, our result showed that the magnetic moment gradually decreased even below $2.5 \mathrm{~K}$. Furthermore, the concentration of paramagnetic impurities in our sample was significantly less than that of pure DPPH.

The difference between the observed magnetic moment and the theoretical curve below around $6 \mathrm{~K}$ suggests that the magnetic behavior of reagent $\mathrm{B}$ at low temperatures did not solely originate from the isolated pair model. In the cases of DPPH-benzene (1:1) below $0.3 \mathrm{~K}$ and $\mathrm{DPPH}-\mathrm{CCl}_{4} \quad(4: 1)$ complex below $0.3 \mathrm{~K}$ exhibited temperature-independent positive magnetic susceptibilities. Consequently, the possibility of inter-pair interaction among nitrogen radicals was discussed. ${ }^{40}$ Using only the result of Fig. 5, it is difficult to judge whether the magnetic moment around $2 \mathrm{~K}$ is intrinsic to DPPH or is influenced by impurities with free radicals.

The X-band EPR spectra taken at a temperature range of 1.5 to $3 \mathrm{~K}$ as shown in Fig. 6 suggests that the magnetic property around $1.8 \mathrm{~K}$ may be intrinsic. Moreover, the magnetic moment measured using the SQUID is a combination of paramagnetic and diamagnetic effects (and others), while the EPR results reveal paramagnetic electrons. The value of $g_{\text {eff }}$ remained constant over the temperature range of $50 \mathrm{~K}$ to room temperature. The inset graph in Fig. 6 shows a plot of peak area versus temperature, where the peak areas were obtained by doubleintegration of each spectrum. Paramagnetic impurities would 
increase the peak area with decreasing $T$ according to the Curie-Weiss law effect. However, the peak area decreased with decreasing $T$. Furthermore, the extrapolation below $1.5 \mathrm{~K}$ showed that the peak area becomes zero around $0.1 \mathrm{~K}$. The peak areas in Fig. 6 may correspond to the number of nitrogen free radicals of DPPH molecules without any interactions. Consequently, the number must decrease with decreasing temperature as the singlet-pairs are created. In addition, the $\mathrm{X}$-band EPR spectrum taken at $1.5 \mathrm{~K}$ over a wide sweep field range of 150 to $390 \mathrm{mT}$ did not detect any peak that indicated paramagnetic impurities.

Therefore, the X-, Q- and W-band EPR measurements did not detect any impurities with free radicals. In addition, trace amounts of impurities with free radicals in reagent B would not result in an error for the purity analysis based on free radical content.

\section{Magnetic properties of reagents $A$ and $C$}

The results of the NMR spectroscopy, CHN analysis, and Q-band EPR measurement suggest that reagents $\mathrm{A}$ and $\mathrm{C}$ include significant amounts of benzene in the DPPH crystal structure. However, the magnetic moments of reagents $\mathrm{A}$ and $\mathrm{C}$ in Fig. 1 being monotonously proportional to $(T-\Theta)^{-1}$ is yet to be explained. Furthermore, the reason several mol\% of benzene suppresses the formation of singlet-state even at liquid $\mathrm{He}$ temperature remains unresolved. If singlet-state pure DPPH at lower temperatures follows only the isolated pair model, nitrogen radicals distant from benzene molecules should still form singlet-pairs and not be completely suppressed as observed. The difference in temperature dependence between pure DPPH and DPPH contaminated by benzene molecules may suggest existence of the inter-pair interaction over a long distance that forms singlet pairs in pure DPPH crystal. The three-axis anisotropic peaks of the W-band spectra in Fig. 4 may be attributed to several mol\% of benzene molecules affecting the localized magnetic environment that surrounds the nitrogen radicals. Furthermore, the additional single peaks detected at the same position as pure DPPH might be due to the existence of free nitrogen radicals away from the benzene molecules in the major component and/or due to free nitrogen radicals of pure DPPH molecules as a minor component. The anisotropic peaks are much higher than the additional peak in the spectrum of reagent $\mathrm{A}$ and $\mathrm{C}$. This indicates that only several $\mathrm{mol} \%$ of benzene may affect magnetic environments surrounding the majority of the nitrogen radicals in each reagent. This change results in a much smaller number of free radicals in reagents $\mathrm{A}$ and $\mathrm{C}$, than that in reagent $\mathrm{B}$.

\section{Conclusion}

Based on free radical content, the result of effective magnetic moment method suggests that only one of the three reagents was uncontaminated with a measured purity of almost $1 \mathrm{~kg} \mathrm{~kg}^{-1}$ with its relative expanded uncertainty of 3.6\%. Expectedly, the use of this reagent as a standard sample for measuring the number of free radicals yields more accurate analytical values. However, such application is delimited at temperatures below $50 \mathrm{~K}$ because the reagent then deviates from the Curie-Weiss law due to the formation of singlet-pairs of nitrogen radicals. On the other hand, the other two reagents exhibited monotonous increasing magnetic moment with decreasing temperature from 300 to $4.2 \mathrm{~K}$ because several mol\% of benzene molecules in the DPPH crystal structure may disturb the formation of the singletpairs. The number of free radicals per unit mass for the two reagents was $20 \%$ less than that of the uncontaminated reagent. This fact may influence analytical results by the EPR spectrometer or the UV/visible spectrometer using the DPPH reagents as the standard sample. The two reagents may not be advisable for use as a standard sample for sweeping field at high-frequency EPR measurements because anisotropic peaks were observed in their Q- and W-band EPR spectra.

\section{Acknowledgements}

A major part of this work was conducted at the Institute for Molecular Science, National Institutes of Natural Sciences, supported by the Nanotechnology Platform Program (Molecule and Material Synthesis) of the Ministry of Education, Culture, Sports, Science and Technology (MEXT), Japan. Part of the SQUID measurements was conducted at the AIST NanoProcessing Facility, supported by the Nanotechnology Platform Program of the same ministry. The authors would like to thank Dr. K. Yamazaki (AIST) for the NMR measurements. An Excel macro program formulated by Dr. T. Shimosaka (AIST) was used for data fitting of magnetic moment via least-squared method. This work was supported by JSPS KAKENHI Grant Number JP17K05918.

\section{Supporting Information}

The following experimental results are presented: (1) modified residual sum of the weighted squared deviation to determine appropriate analytical temperature range based on the CurieWeiss law, (2) temperature dependence of line-width on the X-band EPR measurements and ratio of two-type line-widths, (3) Q-band EPR spectra at room temperature (4) mid-IR spectra at room temperature, and (5) ${ }^{1} \mathrm{H}-\mathrm{NMR}$ spectra when each powder sample was dissolved in DMSO- $d_{6}$. This material is available free of charge on the Web at http://www.jsac.or.jp/ analsci/.

\section{References}

1. D. A. Skoog, D. M. West, F. J. Holler, and S. R. Crouch, "Analytical Chemistry: An Introduction", 7th ed., 2000, Saunders College Publishing, A Division of Harcourt College Publishers, Orlando, FL.

2. M. J. T. Milton and T. J. Quinn, Metrologia, 2001, 38, 289.

3. N. Matsumoto and K. Kato, Metrologia, 2012, 49, 530.

4. N. Matsumoto and T. Shimosaka, Accred. Quality Assur., 2015, 20, 115.

5. N. Matsumoto and T. Shimosaka, J. Appl. Phys., 2015, 117, 17E114.

6. N. Matsumoto and T. Shimosaka, Anal. Sci., 2017, 33, 1059.

7. N. Matsumoto, Bunseki, 2016, 63.

8. T. Kikuchi, K. Kikugawa, and T. Kato, Chem. Pharm. Bull., 1980, 28, 2089.

9. W. Brand-Williams, M. E. Cuvelier, and C. Berset, Food Sci. Technol LWT, 1995, 28, 25.

10. D. Sanna, G. Delogu, M. Mulas, M. Schirra, and A. Fadda, Food Anal. Methods, 2012, 5, 759.

11. E. V. Piletska, S. S. Piletsky, M. J. Whitcombe, I. Chianella, and S. A. Piletsky, Anal. Chem., 2012, 84, 2038.

12. P. Nagaraja, N. Aradhana, A. Suma, A. Shivakumar, and N. A. Chamaraja, Anal. Sci., 2014, 30, 251. 
13. T. Shimamura, Y. Sumikura, T. Yamazaki, A. Tada, T. Kashiwagi, H. Ishikawa, T. Matsui, N. Sugimoto, H. Akiyama, and H. Ukeda, Anal. Sci., 2014, 30, 717.

14. I. Nakanishi, K. Ohkubo, K. Imai, M. Kamibayashi, Y. Yoshihashi, K. Matsumoto, K. Fukuhara, K. Terada, S. Itoh, T. Ozawa, and S. Fukuzumi, Chem. Commun., 2015, 51,8311 .

15. D. Li, J. Jiang, D. Han, X. Yu, K. Wang, S. Zang, D. Lu, A. Yu, and Z. Zhang, Anal. Chem., 2016, 88, 3885.

16. L. Wang, W. Ma, S. Gan, D. Han, Q. Zhang, and L. Niu, Anal. Chem., 2014, 86, 10171.

17. D. B. Hunsaker, Jr. and G. H. Schenk, Talanta, 1983, 30, 475.

18. The Society of Electron Spin Science and Technology, "Introduction to Electron Spin Science and Technology", 2010, Chap. 5, Sangyo-tosho Co. Ltd., Tokyo.

19. K. Komaguchi, T. Maruoka, H. Nakano, I. Imae, Y. Ooyama, and Y. Harima, J. Phys. Chem. C, 2010, 114, 1240.

20. K. Nakagawa, S. Minakawa, D. Sawamura, and H. Hara, Anal. Sci., 2017, 33, 1357.

21. J. Krzystek, A. Sienkiewicz, L. Pardi, and L. C. Brunel, J. Mag. Reson., 1997, 125, 207.

22. B. Cage, A. Weekley, L. C. Brunel, and N. S. Dalal, Anal. Chem., 1999, 71, 1951.

23. R. Miyamoto, M. Iwaki, H. Mino, J. Harada, S. Itoh, and H. Oh-oka, Biochemistry, 2006, 45, 6306.

24. B. Rakvin, D. Žilić, N. S. Dalal, J. M. North, P. Cevc, D. Arčon, and K. Zadro, Spectrochim. Acta, Part A, 2004, 60, 1241.

25. H. Takahashi, T. Okamoto, E. Ohmichi, and H. Ohta, Appl. Phys. Express, 2016, 9, 126701.

26. M. Ikeya, Anal. Sci., 1989, 5, 5.

27. M. Furusawa and M. Ikeya, Anal. Sci., 1988, 4, 649.

28. J. P. Campbell, J. T. Ryan, P. R. Shrestha, Z. Liu, C. Vaz, J-H. Kim, V. Georgiou, and K. P. Cheung, Anal. Chem., 2015, 87, 4910.

29. H. Hirata, T. Kuyama, M. Ono, and Y. Shimoyama, J. Mag. Reson., 2003, 164, 233.

30. T. Suzuki, J. Mag. Reson., 2015, 259, 95.

31. ISO Guide 35-2006, "Reference Materials-General and Statistical Principles for Certification", 3rd ed., 2006, International Organization for Standardization, Switzerland, 31.

32. R. T. Weidner and C. A. Whitmer, Phys. Rev., 1953, 91, 1279.
33. D. E. Williams, J. Am. Chem. Soc., 1967, 89, 4280.

34. N. Ohigashi and H. Inokuchi, Bull. Chem. Soc. Jpn., 1969, 42, 1212.

35. T. Fujito, T. Enoki, H. Ohya-Nishiguchi, and Y. Deguchi, Chem. Lett., 1972, 557.

36. B. N. Misra and S. K. Gupta, Bull. Chem. Soc. Jpn., 1973, 46, 3067.

37. R. Verlinden, P. Grobet, and L. Van Gerven, Chem. Phys. Lett., 1974, 27, 535.

38. T. Yoshioka, H. Ohya-Nishiguchi, and Y. Deguchi, Bull. Chem. Soc. Jpn., 1974, 47, 430.

39. W. Duffy, D. L. Strandburg, and J. F. Deck, J. Chem. Phys., 1978, 68, 2097.

40. T. Fujito, Bull. Chem. Soc. Jpn., 1981, 54, 3110.

41. N. D. Yodanov and A. Christova, Appl. Magn. Reson., 1993, 6, 341.

42. N. D. Yodanov, Appl. Magn. Reson., 1996, 10, 339.

43. D. Žilić, D. Pajić, M. Jurić, K. Molćanov, B. Rakvin, P. Planinić, and K. Zadro, J. Magn. Reson., 2010, 207, 34.

44. N. Itoh, T. Yamazaki, A. Sato, M. Numata, and A. Takatsu, Anal. Sci., 2014, 30, 471.

45. N. Itoh, A. Sato, T. Yamazaki, M. Numata, and A. Takatsu, Anal. Sci., 2013, 29, 1209.

46. H. Fisher, SpringerMaterials, 2.2.2 Nitrogen radicals, Landolt-Börnstein-Group II Molecules and Radicals 1 (Magnetic Properties of Free Radicals), ed. K.-H. Hellwege and A. M. Hellwege, 1965, Chapter DOI:10.1007/10201179_9, Springer-Verlag, Berlin Heidelberg.

47. P. H. Rieger, "Electron Spin Resonance: Analysis and Interpretation", 2007, RSC Publishing, Cambridge, 102.

48. C. Corvaja, "Electron Paramagnetic Resonance: A Practitioner's Toolkit", ed. M. Burustolon and E. Giamello, 2009, John Wiley \& Sons, Inc., NJ.

49. M. Kohno, "Electron Spin Resonance (in Japanese)", 2003, Chap. 3, Ohmsha.

50. SDBSWeb: http://sdbs.db.aist.go.jp (National Institute of Advanced Industrial Science and Technology, Jan 2018).

51. R. M. Silverstein, G. C. Bassler, and T. C. Morrill, "Spectroscopic Identification of Organic Compounds", 5th ed., 1991, Chap. 3, John Wiley \& Sons, Inc., New York.

52. F. Gerson and W. Huber, "Electron Spin Resonance Spectroscopy for Organic Radicals", 2003, Wiley-VCH Verlag GmbH \& Co. KGaA, Weinheim, 296.

53. NIST Chemistry WebBook: Compilation prepared by the National Institute of Standard and Technology, http:// webbook.nist.gov/chemistry/. 